INPLASY

PROTOCOL

To cite: Han et al. Effects of prenatal care on improving anxiety and quality of life in patients with pregnancyinduced hypertension syndrome: A systematic review and meta-analysis. Inplasy protocol 202220037. doi: 10.37766/inplasy2022.2.0037

Received: 12 February 2022

Published: 12 February 2022

Corresponding author: Lingling Han

1377139180@qq.com

Author Affiliation: Basic Medical College of Chengdu University of traditional Chinese Medicine.

Support: None.

Review Stage at time of this submission: Preliminary searches.

Conflicts of interest: None declared.

\section{Effects of prenatal care on improving anxiety and quality of life in patients with pregnancy-induced hypertension syndrome: A systematic review and meta-analysis}

Han, L1; Bai, Y2; Tang, X3; Zhang, Y4; Mo, J5.

Review question / Objective: To evaluate the effect of prenatal care on improving anxiety and quality of life in patients with hypertensive pregnancy syndrome.

Condition being studied: Gestational hypertension syndrome often occurs in pregnant women after 20 weeks of pregnancy, with edema, elevated blood pressure as the main performance in the early stage, gradually aggravated symptoms, headache, nausea, vomiting and other symptoms, serious can appear convulsions, coma symptoms. If not treated in time, it will cause adverse effects on the health of pregnant women and fetuses. In addition, patients with gestational hypertension syndrome have anxiety and other adverse emotions that affect their normal life and reduce their quality of life due to repeated attacks of the disease. Antenatal nursing intervention is to popularize the intervention mode that uses in the light of pregnant high disease patient, this nursing intervention mode emphasizes the intervention to the disease itself in the specific implementation process, still pay attention to physiology and psychological intervention, through reasonable nursing intervention significantly alleviate the anxiety of puerpera and improve puerpera quality of life.

INPLASY registration number: This protocol was registered with the International Platform of Registered Systematic Review and Meta-Analysis Protocols (INPLASY) on 12 February 2022 and was last updated on 12 February 2022 (registration number INPLASY202220037).

\section{INTRODUCTION}

Review question / Objective: To evaluate the effect of prenatal care on improving anxiety and quality of life in patients with hypertensive pregnancy syndrome.

Condition being studied: Gestational hypertension syndrome often occurs in 
pregnant women after 20 weeks of pregnancy, with edema, elevated blood pressure as the main performance in the early stage, gradually aggravated symptoms, headache, nausea, vomiting and other symptoms, serious can appear convulsions, coma symptoms. If not treated in time, it will cause adverse effects on the health of pregnant women and fetuses. In addition, patients with gestational hypertension syndrome have anxiety and other adverse emotions that affect their normal life and reduce their quality of life due to repeated attacks of the disease. Antenatal nursing intervention is to popularize the intervention mode that uses in the light of pregnant high disease patient, this nursing intervention mode emphasizes the intervention to the disease itself in the specific implementation process, still pay attention to physiology and psychological intervention, through reasonable nursing intervention significantly alleviate the anxiety of puerpera and improve puerpera quality of life.

\section{METHODS}

Participant or population: Hypertensive syndrome of pregnancy.

Intervention: Antenatal care.

Comparator: Routine nursing.

Study designs to be included: Randomized controlled trials(RCTs).

Eligibility criteria: Patients with hypertensive pregnancy syndrome (Diagnosed by a clinician) will be included.

Information sources: The databases of CNKI, Wanfang, VIP, Pubmed, Embase and Cochrane Library will be systematically searched for relevant information from the date of establishment to February 2022. Language is limited with English and Chinese.

Main outcome(s): (1)Anxiety, which is measured by Self-Rating Anxiety Scale(SAS) or other tools.(2) Quality of
life(QoL)which is assessed by 36 -Item Short Form Health Survey or other relevant scales.

Quality assessment / Risk of bias analysis: According to the guidance of the Cochrane Handbook for Systematic Review of Interventions Two researcher will assess the risk of bias for each eligible trial from 7 aspects and 3 levels independently. The seven aspects of the risk of bias include Random sequence generation,Allocation concealment,Blinding of participants and personnel,Blinding of outcome assessment ,Incomplete outcome data,Selective reporting and Other bias .Each aspect is further divided as 3 different levels: high, unclear, or low risk of bias.Any disagreements will be resolved via discussion with a third researcher

Strategy of data synthesis: RevMan5.2 software was used to process the extracted data. Relative risk (RR) was used to describe dichotomous variables, mean difference (MD) was used to describe continuous variables, and $95 \%$ confidence interval $(\mathrm{Cl})$ was used to describe the interval estimation of outcome index data. The heterogeneity between studies was tested by $Q$ and 12 statistics. When $P>0.1$ and $12<50 \%$, a fixed effects model was used for the meta-analysis; otherwise, a random effects model was used.

Subgroup analysis: In order to find the source of heterogeneity, the sample size, age and gestational age of each study were used as independent variables for subgroup analysis. If the data is insufficient, qualitative synthesis will be conducted; otherwise quantitative synthesis will be used.

Sensitivity analysis: Sensitivity analysis was conducted by using shear and complement method and single removal method to evaluate the stability of meta-analysis.

Country(ies) involved: China.

Keywords: hypertensive pregnancy syndrome; prenatal care; anxiety; quality of life. 
Contributions of each author:

Author 1 - Lingling Han.

Author 2 - Yiling Bai.

Author 3 - Huiling Tang.

Author 4 - Yiyi Zhang.

Author 5 - Jingwen Mo. 